\title{
Macrozoobenthos of Basti Damar Stream in Rudraprayag District, Garhwal, Uttarakhand: Diversity and Habitat Analysis
}

\author{
Deeksha Mamgain $^{1}$ • Pankaj Bahuguna ${ }^{2}$ Anoop Kumar Dobriyal ${ }^{3} \cdot$ Rajesh Rayal $^{1}$ \\ ${ }^{1}$ Department of Zoology, School of Basic and Applied Sciences, SGRR University, Patel Nagar, Dehradun-248001, \\ Uttarakhand, India. \\ ${ }^{2}$ Biodiversity Lab, Department of Zoology, B. D. Govt. P.G. College Jaiharikhal-246193, Pauri Garhwal, \\ Uttarakhand, India. \\ ${ }^{3}$ Department of Zoology, H. N. B. Garhwal University (A Central University), B. G. R. Campus Pauri, Pauri \\ Garhwal- 246001, Uttarakhand, India.
}

Corresponding Author Email id: drrajeshrayal@gmail.com

Received: 11.04.2021; Revised: 16.06.2021; Accepted: 19.06.2021

(C)Society for Himalayan Action Research and Development

\begin{abstract}
Basti Damar gad is a spring fed tributary of river Mandakini which is quite conducive for biodiversity. As the stream was unexplored, it was taken as an opportunity to investigate the stream for its habitat quality and diversity of benthic organisms. Stream was studied at two different spots one in more or less rhithron zone and the other one in near potamon zone during the year 2019-20. Comparatively, the Spot No 2 had more density (836 individules. $\mathrm{m}^{-2}$ ) and diversity (28genus) of macrozoobenthos than spot No 1 (127 individules. $\mathrm{m}^{-2}$ and 28 genus) which may be due to better substratum heterogeneity dominated by mixed forest, small gravels, small bolder, low water velocity, high water temperature, low canopy of stream area and good survival rate of periphyton on bottom in the stream. Also the water quality at this spot (comparatively low current velocity, moderate temperature, good amount of DO and high alkalinity and $\mathrm{pH}$ ) was supportive for flourishing biota. The observations were statistically analysed using coefficient of correlation, coefficient of determination, regression, species similarity, dominance and diversity indices.
\end{abstract}

Keywords: Macrozoobenthos • Basti Damar Stream • Diversity • Habitat ecology

\section{Introduction}

Biodiversity is a prominent attribute of the aquatic ecosystem for maintaining its stability and a mean of coping with any environmental change (Vinson and Hawkins 1998). In fluvial system, the macrozoobenthos are an important community that dwells at the bottom. Any change in water quality and habitat structure greatly influences the composition, abundance and diversity of these organisms. The macroinvertebrates have also been used in bio-monitoring program that make them particularly beneficial biota (Marzin, 2013). Long term assessment of the population diversity and variability of macrozoobenthos in relation to ecological parameter, especially in the spring fed streams, is required to establish their status, trends and productivity patterns.

The biodiversity baseline data are crucial to develop viable conservation and management strategies (Ward and Tockner 2001). Spring fed streams are the most common water spread throughout Himalayan states in India, because of its beneficial use as potable water, in agriculture and fishery. The conservation of benthic community is essential to maintain the structure and function of these streams as they are an important link of food web for other major biota and also utilize the bottom algae and detritus to make the water clean and suitable for other purposes (Dobriyal, 1985). Fair amount of literature is available on the relationship between 
the macrozoobenthos and environmental variables on the fluvial system in different parts of the world (Resh et.al,1988; Barbour et.al, 1996; Vinson and Hawkins 1998; Jacobson et.al, 2003; Silveira et.al, 2006; Camara et.al. 2012; Uwadiae 2013; Akindele and Liadi 2014; Kabore et.al, 2016) and the Indian sub-continent (Sivaramkrishnan et.al, 1995; Sharma et.al, 2004; Joshi et.al, 2007; Abida et.al, 2012; Negi and Mamgani 2013; Sharma et.al, 2013, Sagir et.al, 2018).

The parent low order streams are the integral and important component of mountain ecosystems of Garhwal Himalayan region. The Bastai Damar is a typical headwater stream and is one of the several tributaries of the Mandakani River in Uttarakhand, India. Fast flowing water and low temperature with high dissolved oxygen characterizes these streams. Monitoring of aquatic insects can provide important insights into changes in stream quality (Rosenberg and Resh 1993). Notable work on substratum heterogeneity and ddistribution pattern of benthic macroinvertebrate community in the various spring fed stream of Garhwal Himalaya in Uttarakhand has been carried out by Dobriyal, et.al. (2009, 2011), Rautela et.al. (2006), Bahuguna and Dobriyal (2018), Balodi and Koshal (2015), Koshal et al (2017) Bahuguna and Negi (2018). The drifting behavioral studies on aquatic insects are also conducted by Bahuguna et.al. $(2019,2020)$. The aim of the present study is to depict the composition and variety of the macrozoobenthos in Basti Damar stream and their inter relations with habitat characteristics.

\section{Material And Methods}

Study area: Basti damaar Gaad is an important spring fed streams originating from the near Badhani Taal peak (Latitude 30.49 85'94" E, Longitude $\left.78.91^{\circ} 94^{\prime} 32^{\prime \prime} \quad \mathrm{N}\right)$ in districtRudraprayag from Garhwal Himalaya.

Physico-chemical analysis: The physicochemical parameters of the stream were conducted on two spots as per standard Method recommended by APHA (2005). The parameters investigated includes water temperature, current velocity, dissolved oxygen, $\mathrm{pH}$, free $\mathrm{CO}_{2}$, total Alkalinity and total hardness.

\section{Macrozoobenthos Sampling}

(a) Collection and preservation of macrozoobenthos: Macrozoobenthos were collected with the help of Surber sampler of one feet $^{2}$ area and preserved in suitable preservatives (4\% Formalin or alcohol as desired)..

(b) Taxonomy of Macrozoobenthos: Macrozoobenthos were identified to genus level in common and up to species level wherever possible) with the help of available identification keys (Ward and Whipple, 1959; Needham and Needham, 1962; Hynes, 1977; Elliott, et.al., 1988; Wallace, et.al., 2003; Bouchard, et.al., 2012; etc.).

(c) Diversity studies: Statistical analysis of the data was made with the help of PAST statistical software. Common statistical tools used were regression, correlation, similarity and diversity indices (Shannon and Wiener, Margalef, any other suitable indices).

\section{Results and Discussion}

Data related to density and diversity of macrozoobenthos in Basti damr stream at Spot no 1, sampled during two year period is presented in Table 1

It shows that a total of five orders of macrozoobenthos were recorded at this spot comprising 12 different genera. The maximum diversity was seen in order Ephemeroptera with highest of 4 genus and rest all 4 orders have 2 genus each. Highest density was observed in the month of December (127 individuals. $\left.\mathrm{m}^{-2}\right)$ followed by November (117 individuals. $\mathrm{m}^{-2}$ ) and October (114 individuals. $\left.\mathrm{m}^{-2}\right)$ and lowest in the month of August (17 individuals. $\mathrm{m}^{-2}$ ) followed by July (70 individuals. $\mathrm{m}^{-2}$ ). From the recorded data it is evident that density is maximum in winter season (i.e. 321 individuals) and minimum in Monsoon season (87 individuals) whereas diversity was maximum in the month of April and December where individuals from all 12 genus were recorded and minimum in the month of August with least of 8 genus (Table 1-3). 
The diversity at Spot 2 is more than Spot 1. A total of 28 genus was recorded here. The maximum diversity was noticed in Ephemeroptera with 8 genus followed by order Trichoptera and Diptera with 5 genus each, Coleoptera with 4 , Odonata with 3, Hemiptera with 2 genus and minimum 1 genus in Plecoptera (Table 4-6). Plecoptera and Hemiptera were two orders found only at Spot 2 with 3 genus. The highest number of individuals were observed in the month of December (836 individuals $\mathrm{m}^{-2}$ ), January (736 individuals $\mathrm{m}^{-2}$ ) and November (729 individuals $\mathrm{m}^{-2}$ ) and least in the month of August with only 37 individuals $\mathrm{m}^{-2}$ in total from 15 genus. The diversity was observed maximum in the month of April, June and November represented by the individuals of all 28 genus and minimum in the month of August with individuals from only 15 genus.

Genus Baeties, Caenis, Ecdyonurus, Heptagenia, Hydropsyche, Stenopsyche, Simulium, Tipula, Psephenus, Hydrophilus, Argia and Euphaea were recorded from both the spots whereas Ameletus, Atalophlebie, Ephemerella, Isonychia, Chimarra, Glossosoma, Rhyacophila, Berosous, Elmid, Antocha, Atherix, Chironomus, Zygonyx, Neoperla, Gerris and Heleocoris were recorded only from the Spot 2 during the entire course of study. During the monsoon density was observed minimum with a total of only 242 individuals $\mathrm{m}^{-2}$ whereas maximum was noticed during winters with a total of 2238 individuals $\mathrm{m}^{-2}$

Data related to density and diversity of macrozoobenthos in Basti damer stream at Spot no 2, sampled during two year period is presented in Table 4.

Species dominance and diversity indices for macrozoobenthos were presented in the Table 7 and 8 for sampling spots 1 and 2 respectively.
The maximum diversity was observed in order Ephemeroptera with highest of 4 genus and rest all 4 orders have 2 genus each in spot- 1 . For spot-2, the highest diversity was estimated in order Ephemeroptera with 8 genus followed by order Trichoptera and Diptera with 5 genus each, Coleoptera with 4 , Odonata with 3 , Hemiptera with 2 genus and lowest with 1 genus in Plecoptera. In basti Damar stream, Ephemeroptera were dominant taxa in both the spots. Ephemeroptera (may flies) was also reported dominant taxon in the Khanda Gad by Kumar And Dobriyal (1996) and in Gaula gad of Kumaun region by Sunder (1997). Bahuguna and Negi (2018) also noticed that Ephemeroptera were dominant taxa in Kyunja Gad stream, a spring-fed tributary of river Mandakani. In this study, Trichoptera and Diptera were other dominant taxa in both spots. Nautiyal et al. (2015) noticed that Trichopterans were main taxa in the headwater section of the lesser Himalayan spring-fed streams.

Physico-chemical parameters are presented in the Table 9 and 10 for the Spot-1 and Spot-2 respectively. 
Table 1: Macrozoobenthos density (units. $\mathrm{m}^{-2}$ ) and diversity during April 2019 to March 2020 from Basti damaar Gad stream (Spot-1).

\begin{tabular}{|c|c|c|c|c|c|c|c|c|c|c|c|c|c|}
\hline $\mathbf{S N}$ & $\begin{array}{c}\text { Name of the } \\
\text { Order/Genus/Species }\end{array}$ & Apr & May & Jun & Jul & Aug & Sep & Oct & Nov & Dec & Jan & Feb & Mar \\
\hline $\mathbf{A}$ & \multicolumn{13}{|c|}{ EPHEMEROPTERA (units.m ${ }^{-2}$ ) } \\
\hline 1 & Baeties & 10 & 16 & 15 & 14 & 2 & 13 & 15 & 13 & 10 & 12 & 15 & 14 \\
\hline 2 & Caenis & 5 & 9 & 12 & 10 & 3 & 7 & 5 & 6 & 8 & 5 & 9 & 12 \\
\hline 3 & Ecdyonurus & 3 & 1 & 5 & 2 & 1 & 1 & 2 & 8 & 5 & 2 & 3 & 5 \\
\hline \multirow[t]{2}{*}{4} & Heptagenia & 15 & 17 & 16 & 15 & 1 & 12 & 13 & 10 & 17 & 10 & 13 & 16 \\
\hline & TOTAL & 33 & 43 & 48 & 41 & 7 & 33 & 35 & 37 & 40 & 29 & 40 & 47 \\
\hline $\mathbf{B}$ & \multicolumn{13}{|c|}{ TRICHOPTERA（units.m ${ }^{-2}$ ) } \\
\hline 1 & Hydropsyche & 28 & 25 & 15 & 5 & 7 & 14 & 20 & 24 & 18 & 21 & 15 & 32 \\
\hline \multirow[t]{2}{*}{2} & Stenopsyche himalayana & 4 & 3 & 2 & 2 & 1 & 6 & 10 & 2 & 2 & 3 & 2 & 1 \\
\hline & TOTAL & 32 & 28 & 17 & 7 & 8 & 20 & 30 & 26 & 20 & 24 & 17 & 33 \\
\hline $\mathbf{C}$ & \multicolumn{13}{|l|}{ DIPTERA (units.m ${ }^{-2}$ ) } \\
\hline 1 & Simulium & 16 & 15 & 15 & 15 & 1 & 13 & 25 & 24 & 27 & 19 & 18 & 17 \\
\hline \multirow[t]{2}{*}{2} & Tipula & 3 & 4 & 3 & 0 & 0 & 3 & 2 & 1 & 5 & 0 & 0 & 0 \\
\hline & TOTAL & 19 & 19 & 18 & 15 & 1 & 16 & 27 & 25 & 32 & 19 & 18 & 17 \\
\hline $\mathbf{D}$ & \multicolumn{13}{|c|}{ COLEOPTERA (units.m ${ }^{-2}$ ) } \\
\hline 1 & Psephenus tenulpes & 15 & 16 & 18 & 5 & 0 & 15 & 20 & 24 & 28 & 22 & 20 & 14 \\
\hline \multirow[t]{2}{*}{2} & Hydrophilus & 2 & 1 & 0 & 0 & 1 & 1 & 0 & 2 & 2 & 0 & 0 & 1 \\
\hline & TOTAL & 17 & 17 & 18 & 5 & 1 & 16 & 20 & 26 & 30 & 22 & 20 & 15 \\
\hline $\mathbf{E}$ & \multicolumn{13}{|l|}{ ODONATA (units.m $\left.{ }^{-2}\right)$} \\
\hline 1 & Argia & 3 & 0 & 2 & 1 & 0 & 2 & 0 & 0 & 1 & 0 & 0 & 0 \\
\hline \multirow[t]{2}{*}{2} & Euphaea & 1 & 0 & 3 & 1 & 0 & 0 & 2 & 3 & 4 & 2 & 3 & 2 \\
\hline & TOTAL & 4 & $\mathbf{0}$ & 5 & 2 & $\mathbf{0}$ & 2 & 2 & 3 & 5 & 2 & 3 & 2 \\
\hline \multicolumn{2}{|c|}{ TOTAL NO. OF SPECIES } & 105 & 107 & 106 & 70 & 17 & 87 & 114 & 117 & 127 & 96 & 98 & 114 \\
\hline
\end{tabular}

Table 2: Order wise population of Macrozoobenthos (units. $\left.\mathrm{m}^{-2}\right)$ at Spot no. 1 ( $1^{\text {st }}$ order stream)

\begin{tabular}{lcccccccccccc}
\hline Order & Apr. & May & Jun. & Jul. & Aug. & Sep. & Oct. & Nov. & Dec. & Jan. & Feb. & Mar. \\
\hline Ephemeroptera & 33 & 43 & 48 & 41 & 7 & 33 & 35 & 37 & 40 & 29 & 40 & 47 \\
Trichoptera & 32 & 28 & 17 & 7 & 8 & 20 & 30 & 26 & 20 & 24 & 17 & 33 \\
Diptera & 19 & 19 & 18 & 15 & 1 & 16 & 27 & 25 & 32 & 19 & 18 & 17 \\
Coleoptera & 17 & 17 & 18 & 5 & 1 & 16 & 20 & 26 & 30 & 22 & 20 & 15 \\
Odonata & 4 & 0 & 5 & 2 & 0 & 2 & 2 & 3 & 5 & 2 & 3 & 2 \\
Total & $\mathbf{1 0 5}$ & $\mathbf{1 0 7}$ & $\mathbf{1 0 6}$ & $\mathbf{7 0}$ & $\mathbf{1 7}$ & $\mathbf{8 7}$ & $\mathbf{1 1 4}$ & $\mathbf{1 1 7}$ & $\mathbf{1 2 7}$ & $\mathbf{9 6}$ & $\mathbf{9 8}$ & $\mathbf{1 1 4}$ \\
\hline
\end{tabular}

Table 3: Season wise population of Macrozoobenthos (units.m $\left.{ }^{-2}\right)$ at Spot no. 1 ( $1^{\text {st }}$ order stream)

\begin{tabular}{lccccc}
\hline Order & $\begin{array}{c}\text { Spring } \\
\text { (March \& } \\
\text { April) }\end{array}$ & $\begin{array}{c}\text { Summer } \\
\text { (May \& June) }\end{array}$ & $\begin{array}{c}\text { Monsoon } \\
\text { (July \& August) }\end{array}$ & $\begin{array}{c}\text { Autumn (Sept., } \\
\text { Oct. \& Nov.) }\end{array}$ & $\begin{array}{c}\text { Winter (Dec., Jan. } \\
\text { and Feb.) }\end{array}$ \\
\hline Ephemeroptera & 80 & 91 & 48 & 105 & 109 \\
Trichoptera & 65 & 45 & 15 & 76 & 61 \\
Diptera & 36 & 37 & 16 & 68 & 69 \\
Coleoptera & 32 & 35 & 6 & 62 & 72 \\
Odonata & 6 & 5 & 2 & 7 & 10 \\
Total & $\mathbf{2 1 9}$ & $\mathbf{2 1 3}$ & $\mathbf{8 1 8}$ & $\mathbf{3 2 1}$ \\
\hline
\end{tabular}

Table 4: Macrozoobenthos density (units. $\mathrm{m}^{-2}$ ) and diversity during April 2019 to March 2020 from Basti damaar Gad stream (Spot-2) ( $2^{\text {nd }}$ Order Stream).

\begin{tabular}{|c|c|c|c|c|c|c|c|c|c|c|c|c|c|}
\hline S.No. & $\begin{array}{l}\text { Name of the } \\
\text { Order/Genus/Species }\end{array}$ & Apr. & May & Jun. & Jul. & Aug. & Sep. & Oct. & Nov. & Dec. & Jan. & Feb. & Mar. \\
\hline $\mathbf{A}$ & EPHEMEROPTERA & & & & & & & & & & & & \\
\hline 1 & Ameletus & 20 & 19 & 15 & 0 & 4 & 19 & 25 & 24 & 31 & 28 & 27 & 24 \\
\hline
\end{tabular}




\begin{tabular}{|c|c|c|c|c|c|c|c|c|c|c|c|c|c|}
\hline 2 & Atalophlebie & 7 & 5 & 3 & 11 & 0 & 4 & 3 & 4 & 7 & 5 & 8 & 6 \\
\hline 3 & Baeties & 42 & 35 & 22 & 8 & 0 & 36 & 55 & 42 & 59 & 51 & 49 & 48 \\
\hline 4 & Caenis & 5 & 6 & 15 & 10 & 0 & 3 & 5 & 9 & 19 & 14 & 14 & 2 \\
\hline 5 & Ecdyonurus & 2 & 1 & 1 & 2 & 1 & 1 & 0 & 2 & 3 & 7 & 1 & 4 \\
\hline 6 & Ephemerella indica & 2 & 1 & 2 & 1 & 0 & 2 & 2 & 1 & 3 & 1 & 3 & 2 \\
\hline 7 & Heptagenia & 2 & 2 & 1 & 2 & 2 & 2 & 3 & 4 & 6 & 5 & 3 & 2 \\
\hline \multirow[t]{2}{*}{8} & Isonychia & 1 & 0 & 1 & 0 & 0 & 0 & 1 & 1 & 0 & 0 & 0 & 0 \\
\hline & TOTAL & 81 & 69 & 60 & 34 & 7 & 67 & 94 & 87 & 128 & 111 & 105 & 88 \\
\hline $\mathbf{B}$ & TRICHOPTERA & & & & & & & & & & & & \\
\hline 1 & Chimarra & 17 & 16 & 10 & 6 & 1 & 19 & 25 & 30 & 31 & 35 & 26 & 24 \\
\hline 2 & Glossosoma caudatum & 14 & 10 & 7 & 3 & 0 & 9 & 17 & 21 & 29 & 21 & 20 & 17 \\
\hline 3 & Hydropsyche & 89 & 71 & 42 & 20 & 6 & 58 & 109 & 121 & 124 & 108 & 106 & 100 \\
\hline 4 & Rhyacophila similis & 31 & 25 & 16 & 4 & 2 & 21 & 45 & 60 & 72 & 70 & 55 & 49 \\
\hline \multirow[t]{2}{*}{5} & Stenopsyche himalayana & 17 & 21 & 13 & 5 & 2 & 11 & 21 & 18 & 36 & 26 & 19 & 19 \\
\hline & TOTAL & 168 & 143 & 88 & 38 & 11 & 118 & 217 & 250 & 292 & 260 & 226 & 209 \\
\hline $\mathrm{C}$ & COLEOPTERA & & & & & & & & & & & & \\
\hline 1 & Berosous indicus & 8 & 12 & 7 & 1 & 2 & 8 & 19 & 16 & 12 & 10 & 9 & 7 \\
\hline 2 & Elmid(larva) & 12 & 10 & 4 & 0 & 0 & 5 & 8 & 7 & 8 & 9 & 6 & 16 \\
\hline 3 & Hydrophilus & 15 & 14 & 10 & 0 & 0 & 11 & 14 & 18 & 21 & 18 & 14 & 18 \\
\hline \multirow[t]{2}{*}{4} & Psephenus tenulpes & 68 & 51 & 34 & 29 & 5 & 40 & 93 & 140 & 151 & 129 & 102 & 87 \\
\hline & TOTAL & 103 & 87 & 55 & 30 & 7 & 64 & 134 & 181 & 192 & 166 & 131 & 128 \\
\hline D & DIPTERA & & & & & & & & & & & & \\
\hline 1 & Antocha & 1 & 2 & 1 & 0 & 0 & 1 & 2 & 3 & 2 & 4 & 2 & 3 \\
\hline 2 & Atherix & 5 & 4 & 3 & 1 & 0 & 4 & 5 & 3 & 6 & 7 & 5 & 7 \\
\hline 3 & Chironomus & 25 & 21 & 18 & 23 & 2 & 21 & 40 & 58 & 48 & 55 & 42 & 30 \\
\hline 4 & Simulium & 90 & 62 & 29 & 65 & 3 & 51 & 115 & 107 & 123 & 103 & 132 & 114 \\
\hline \multirow[t]{2}{*}{5} & Tipula & 1 & 2 & 2 & 0 & 1 & 1 & 2 & 1 & 3 & 4 & 3 & 2 \\
\hline & TOTAL & 122 & 91 & 53 & 89 & 6 & 78 & 164 & 172 & 182 & 173 & 184 & 156 \\
\hline $\mathbf{E}$ & ODONATA & & & & & & & & & & & & \\
\hline 1 & Argia & 9 & 2 & 2 & 1 & 0 & 7 & 15 & 16 & 19 & 8 & 5 & 8 \\
\hline 2 & Euphaea & 3 & 3 & 2 & 1 & 0 & 3 & 5 & 6 & 7 & 8 & 4 & 2 \\
\hline \multirow[t]{2}{*}{3} & Zygonyx & 3 & 2 & 1 & 2 & 1 & 4 & 3 & 4 & 3 & 2 & 1 & 3 \\
\hline & TOTAL & 15 & 7 & 5 & 4 & 1 & 14 & 23 & 26 & 29 & 18 & 10 & 13 \\
\hline $\mathbf{F}$ & PLECOPTERA & & & & & & & & & & & & \\
\hline \multirow[t]{2}{*}{1} & Neoperla & 2 & 5 & 6 & 7 & 4 & 10 & 5 & 8 & 9 & 6 & 4 & 0 \\
\hline & TOTAL & 2 & 5 & 6 & 7 & 4 & 10 & 5 & 8 & 9 & 6 & 4 & $\mathbf{0}$ \\
\hline G & HEMIPTERA & & & & & & & & & & & & \\
\hline 1 & Gerris & 2 & 2 & 2 & 1 & 0 & 1 & 1 & 3 & 2 & 1 & 2 & 1 \\
\hline \multirow[t]{2}{*}{2} & Heleocoris vicinus & 3 & 2 & 1 & 2 & 1 & 2 & 3 & 2 & 2 & 1 & 4 & 3 \\
\hline & TOTAL & 5 & 4 & 3 & 3 & 1 & 3 & 4 & 5 & 4 & 2 & 6 & 4 \\
\hline \multicolumn{2}{|c|}{ TOTAL NO. OF SPECIES } & 496 & 406 & 270 & 205 & 37 & 354 & 641 & 729 & 836 & 736 & 666 & 598 \\
\hline
\end{tabular}

Table 5: Order wise population of Macrozoobenthos at Spot no. 2 (2 $2^{\text {nd }}$ order stream)

\begin{tabular}{lcccccccccccc}
\hline Order & Apr. & May & Jun. & Jul. & Aug. & Sep. & Oct. & Nov. & Dec. & Jan. & Feb. & Mar. \\
\hline Ephemeroptera & 81 & 69 & 60 & 34 & 7 & 67 & 94 & 87 & 128 & 111 & 105 & 88 \\
Trichoptera & 168 & 143 & 88 & 38 & 11 & 118 & 217 & 250 & 292 & 260 & 226 & 209 \\
Coleoptera & 103 & 87 & 55 & 30 & 7 & 64 & 134 & 181 & 192 & 166 & 131 & 128 \\
Diptera & 122 & 91 & 53 & 89 & 6 & 78 & 164 & 172 & 182 & 173 & 184 & 156 \\
Odonata & 15 & 7 & 5 & 4 & 1 & 14 & 23 & 26 & 29 & 18 & 10 & 13 \\
Plecoptera & 2 & 5 & 6 & 7 & 4 & 10 & 5 & 8 & 9 & 6 & 4 & 0 \\
Hemiptera & 5 & 4 & 3 & 3 & 1 & 3 & 4 & 5 & 4 & 2 & 6 & 4 \\
Total & $\mathbf{4 9 6}$ & $\mathbf{4 0 6}$ & $\mathbf{2 7 0}$ & $\mathbf{2 0 5}$ & $\mathbf{3 7}$ & $\mathbf{3 5 4}$ & $\mathbf{6 4 1}$ & $\mathbf{7 2 9}$ & $\mathbf{8 3 6}$ & $\mathbf{7 3 6}$ & $\mathbf{6 6 6}$ & $\mathbf{5 9 8}$ \\
\hline
\end{tabular}


Table 6: Season wise population of Macrozoobenthos at Spot no. 2 (2 $2^{\text {nd }}$ order stream)

\begin{tabular}{lccccc}
\hline Order & $\begin{array}{c}\text { Spring } \\
\text { (March \& April) }\end{array}$ & $\begin{array}{c}\text { Summer } \\
\text { (May \& June) }\end{array}$ & $\begin{array}{c}\text { Monsoon } \\
\text { (July \& August) }\end{array}$ & $\begin{array}{c}\text { Autumn (Sept., } \\
\text { Oct. \& Nov.) }\end{array}$ & $\begin{array}{c}\text { Winter (Dec., } \\
\text { Jan. and Feb.) }\end{array}$ \\
\hline Ephemeroptera & 169 & 129 & 41 & 248 & 344 \\
Trichoptera & 377 & 231 & 49 & 585 & 778 \\
Coleoptera & 231 & 142 & 37 & 379 & 489 \\
Diptera & 278 & 144 & 95 & 414 & 539 \\
Odonata & 28 & 12 & 5 & 63 & 57 \\
Plecoptera & 2 & 11 & 11 & 23 & 19 \\
Hemiptera & 9 & 7 & 4 & 12 & 12 \\
Total & $\mathbf{1 0 9 4}$ & $\mathbf{6 7 6}$ & $\mathbf{2 4 2}$ & $\mathbf{1 7 2 4}$ & $\mathbf{2 2 3 8}$ \\
\hline
\end{tabular}

Table 7: Dominance and diversity indices of Macrozoobenthos at Spot no. 1 ( $1^{\text {st }}$ order stream) based on PAST

\begin{tabular}{lcccccccccccccccc}
\hline Months & Apr. & May & Jun. & Jul. & Aug. & Sep. & Oct. & Nov. & Dec. & Jan. & Feb. & Mar. \\
\hline Taxa_S & 12 & 10 & 11 & 10 & 8 & 11 & 10 & 11 & 12 & 9 & 9 & 10 \\
Individuals & 105 & 107 & 106 & 70 & 17 & 87 & 114 & 117 & 127 & 96 & 98 & 114 \\
Dominance_D & 0.1508 & 0.1536 & 0.1290 & 0.1645 & 0.2318 & 0.1325 & 0.1505 & 0.1545 & 0.1466 & 0.1706 & 0.1506 & 0.1644 \\
Simpson_1-D & 0.8492 & 0.8464 & 0.871 & 0.8355 & 0.7682 & 0.8675 & 0.8495 & 0.8455 & 0.8534 & 0.8294 & 0.8494 & 0.8356 \\
Shannon_H & 2.113 & 1.994 & 2.159 & 1.962 & 1.757 & 2.131 & 2.021 & 2.039 & 2.115 & 1.91 & 1.99 & 1.959 \\
Evenness_e^H/S & 0.6894 & 0.7348 & 0.7874 & 0.7111 & 0.724 & 0.7658 & 0.7548 & 0.6984 & 0.6911 & 0.7501 & 0.8131 & 0.7094 \\
Margalef & 2.364 & 1.926 & 2.144 & 2.118 & 2.471 & 2.239 & 1.9 & 2.1 & 2.271 & 1.753 & 1.745 & 1.9 \\
\hline
\end{tabular}

Table 8: Dominance and diversity indices of Macrozoobenthos at Spot no. 2 ( $2^{\text {nd }}$ order stream) based on PAST.

\begin{tabular}{|c|c|c|c|c|c|c|c|c|c|c|c|c|}
\hline Months & Apr. & May & Jun. & Jul. & Aug. & Sep. & Oct. & Nov. & Dec. & Jan. & Feb. & Mar. \\
\hline Taxa_S & 28 & 27 & 28 & 22 & 15 & 27 & 27 & 28 & 27 & 27 & 27 & 26 \\
\hline Individuals & 496 & 406 & 270 & 205 & 37 & 354 & 641 & 729 & 836 & 736 & 666 & 598 \\
\hline Dominance_D & 0.105 & 0.094 & 0.080 & 0.153 & 0.092 & 0.088 & 0.0105 & 0.108 & 0.100 & 0.098 & 0.110 & 0.108 \\
\hline Simpson_1-D & 0.895 & 0.905 & 0.919 & 0.846 & 0.907 & 0.941 & 0.894 & 0.891 & 0.899 & 0.901 & 0.889 & 0.891 \\
\hline Shannon_H & 2.614 & 2.68 & 2.796 & 2.329 & 2.527 & 2.724 & 2.578 & 2.565 & 2.627 & 2.625 & 2.545 & 2.552 \\
\hline Evenness_e ${ }^{\wedge} H / S$ & 0.487 & 0.540 & 0.582 & 0.466 & 0.834 & 0.564 & 0.487 & 0.464 & 0.512 & 0.511 & 0.472 & 0.493 \\
\hline Margalef & 4.350 & 4.329 & 4.823 & 3.945 & 3.877 & 4.330 & 4.023 & 4.096 & 3.864 & 3.939 & 3.999 & 3.91 \\
\hline
\end{tabular}

Table 9: Physico-chemical parameters of Basti dammar stream at Spot No 1 (April,2019-March, 2020)

\begin{tabular}{|c|c|c|c|c|c|c|c|c|c|c|c|c|}
\hline Month & Apr. & May & Jun. & Jul. & Aug & Sep. & Oct. & Nov. & Dec. & Jan. & Feb. & Mar. \\
\hline \multirow[t]{2}{*}{ Temp } & 8.75 & 9.2 & 10.70 & 10.85 & 11.55 & 10.75 & 10.2 & 8.55 & 8.2 & 7.35 & 7.7 & 8.4 \\
\hline & \pm 0.07 & \pm 0.14 & \pm 0.10 & \pm 0.07 & \pm 0.07 & \pm 0.07 & \pm 0.14 & \pm 0.40 & \pm 0.14 & $\pm \mathbf{0 . 0 7}$ & \pm 0.14 & \pm 0.14 \\
\hline \multirow[t]{2}{*}{ pH } & 7.55 & 7.65 & 7.55 & 7.45 & 7.25 & 7.55 & 7.6 & 7.55 & 7.6 & 7.75 & 7.55 & 7.8 \\
\hline & \pm 0.07 & \pm 0.07 & \pm 0.07 & \pm 0.07 & \pm 0.07 & \pm 0.07 & \pm 0.14 & \pm 0.07 & \pm 0.00 & \pm 0.07 & \pm 0.07 & \pm 0.14 \\
\hline \multirow[t]{2}{*}{ Velocity } & 0.338 & 0.361 & 0.404 & 0.501 & 0.541 & 0.413 & 0.359 & 0.309 & 0.270 & 0.234 & 0.268 & 0.304 \\
\hline & \pm 0.10 & \pm 0.06 & \pm 0.15 & \pm 0.20 & \pm 0.25 & \pm 0.41 & \pm 0.26 & \pm 0.22 & \pm 0.15 & \pm 0.02 & \pm 0.06 & \pm 0.08 \\
\hline \multirow[t]{2}{*}{ D.O. } & 7.85 & 7.7 & 7.25 & 7.15 & 7.10 & 7.15 & 7.45 & 7.55 & 7.85 & 8.4 & 8 & 7.85 \\
\hline & \pm 0.13 & \pm 0.50 & \pm 0.18 & \pm 0.09 & \pm 0.42 & \pm 0.00 & \pm 0.16 & \pm 0.24 & \pm 0.35 & \pm 0.34 & \pm 0.12 & \pm 0.08 \\
\hline \multirow[t]{2}{*}{ Total Alkalinity } & 92.15 & 93.65 & 86.8 & 81.1 & 80.15 & 82.95 & 84.15 & 86.1 & 87.8 & 90.4 & 89.7 & 92.4 \\
\hline & \pm 2.47 & \pm 1.34 & \pm 0.71 & \pm 1.13 & \pm 0.07 & \pm 0.35 & \pm 0.49 & \pm 0.71 & \pm 0.70 & \pm 0.42 & \pm 0.71 & \pm 0.00 \\
\hline \multirow[t]{2}{*}{ Total Hardness } & 85.1 & 79.3 & 77.2 & 79.55 & 74.8 & 88.05 & 90.3 & 93.1 & 98.95 & 105.7 & 101.7 & 88.7 \\
\hline & \pm 1.70 & \pm 0.14 & \pm 1.27 & \pm 0.92 & \pm 0.85 & \pm 1.13 & \pm 0.28 & \pm 1.13 & \pm 0.92 & \pm 0.71 & \pm 0.85 & \pm 1.13 \\
\hline
\end{tabular}

At spot No 1, the upper reach area had water temperature fluctuations from $7.35 \pm 0.07^{0} \mathrm{C}$ (January) to $11.55 \pm 0.07^{\circ} \mathrm{C}$ (August) and at spot no 2, the lower reach from $9.35 \pm 0.05^{\circ} \mathrm{C}$ (January) to $17.3 \pm 0.14^{0} \mathrm{C}$ (August). $\mathrm{pH}$ fluctuated from 7.25 \pm 0.07 (August) to $7.8 \pm 0.14$ (March) in Spot-1 and 7.5 \pm 0.04 (August) to $7.5 \pm 0.07$ (January) in Spot-2. Current velocity was minimum $0.234 \pm 0.02$ (January) and maximum 0.541 \pm 0.25 (August) 
in Spot-1 and $0.216 \pm 0.04 \mathrm{~m} \cdot \mathrm{s}^{-1}$ (January) to $0.490 \pm 0.05 \mathrm{~m} . \mathrm{s}^{-1}$ (August) in Spot-2. DO values were in a range of $7.10 \pm 0.42$ (August) to $8.4 \pm 0.34$ mg. $\mathrm{l}^{-1}$ (January) in Spot-1 and $7.15 \pm 0.21$ (August) to $8.25 \pm 0.05 \mathrm{mg} . \mathrm{l}^{-1}$ (January) in Spot-2. Total alkalinity was
$80.15 \pm 0.07$ (August) to $90.4 \pm 0.42 \mathrm{mg} . \mathrm{l}^{-1}$ in Spot-1 and 78.75 \pm 0.78 (August) to $96.9 \pm 0.99 \mathrm{mg} . \mathrm{l}^{-1}$ in spot-2 and total hardness fluctuated from $74.8 \pm 0.85$ to $105.7 \pm 0.71 \mathrm{mg} .1^{1}$ ${ }^{1}$ in Spot-1 and $73.15 \pm 1.4$ to $101.3 \pm 4.3 \mathrm{mg} . \mathrm{l}^{-1}$ in Spot-2.

Table 10: Physico-chemical parameters of water at sampling Spot 2 in spring fed stream Basti damaar (April,2019-March, 2020).

\begin{tabular}{|l|c|c|c|c|c|c|c|c|c|c|c|c|}
\hline Month & Apr. & May & Jun. & Jul. & Aug. & Sep. & Oct. & Nov. & Dec. & Jan. & Feb. & Mar. \\
\hline Temperat & 12.7 & 14.15 & 15.7 & 16.2 & $\mathbf{1 7 . 3}$ & 16.65 & 13.7 & 12.05 & 10.2 & $\mathbf{9 . 3 5}$ & 10.1 & 10.80 \\
ure & \pm 0.28 & \pm 0.21 & \pm 0.26 & \pm 0.14 & $\mathbf{\pm 0 . 1 4}$ & \pm 0.21 & \pm 0.28 & \pm 0.07 & \pm 0.14 & $\pm \mathbf{0 . 0 7}$ & \pm 0.14 & \pm 0.12 \\
\hline \multirow{2}{*}{ pH } & 7.35 & 7.3 & 7.45 & 7.25 & $\mathbf{7 . 1 5}$ & 7.35 & 7.45 & 7.45 & 7.45 & $\mathbf{7 . 5}$ & 7.35 & 7.30 \\
& \pm 0.07 & \pm 0.0 & \pm 0.05 & \pm 0.07 & $\mathbf{\pm 0 . 0 4}$ & \pm 0.09 & \pm 0.07 & \pm 0.20 & \pm 0.37 & $\mathbf{\pm 0 . 0 7}$ & \pm 0.05 & \pm 0.00 \\
\hline \multirow{2}{*}{ Velocity } & 0.305 & 0.317 & 0.343 & 0.426 & $\mathbf{0 . 4 9 0}$ & 0.310 & 0.280 & 0.267 & 0.253 & $\mathbf{0 . 2 1 6}$ & 0.242 & 0.260 \\
& \pm 0.01 & \pm 0.05 & \pm 0.02 & \pm 0.04 & $\mathbf{\pm 0 . 0 5}$ & \pm 0.08 & \pm 0.07 & \pm 0.6 & \pm 0.02 & $\mathbf{\pm 0 . 0 4}$ & \pm 0.02 & \pm 0.05 \\
\hline \multirow{2}{*}{ D.O. } & 7.45 & 7.65 & 7.4 & 7.25 & $\mathbf{7 . 1 5}$ & 7.45 & 7.65 & 7.75 & 8.1 & $\mathbf{8 . 2 5}$ & 7.75 & 7.65 \\
& \pm 0.8 & \pm 0.10 & \pm 0.00 & \pm 0.06 & $\mathbf{\pm 0 . 2 1}$ & \pm 0.05 & \pm 035 & \pm 0.50 & \pm 0.80 & $\pm \mathbf{0 . 0 5}$ & \pm 0.07 & \pm 0.15 \\
\hline Total & 91.65 & 88.25 & 86.95 & 81 & $\mathbf{7 8 . 7 5}$ & 82.35 & 84 & 86.95 & 89.7 & $\mathbf{9 6 . 9}$ & 95.75 & 92.7 \\
Alkalinity & \pm 0.64 & \pm 1.48 & \pm 0.64 & \pm 0.71 & $\mathbf{\pm 0 . 7 8}$ & \pm 1.63 & \pm 0.28 & \pm 1.06 & \pm 0.57 & $\mathbf{\pm 0 . 9 9}$ & \pm 0.64 & \pm 0.57 \\
\hline Total & 81.3 & 71.5 & 75.75 & 74.7 & $\mathbf{7 3 . 1 5}$ & 80.8 & 88.5 & 90.5 & 96.25 & $\mathbf{1 0 1 . 3}$ & 97.45 & 84.05 \\
Hardness & \pm 2.9 & \pm 1.7 & \pm 3.2 & \pm 2.8 & $\mathbf{\pm 1 . 4}$ & \pm 2.8 & \pm 4.9 & \pm 3.4 & \pm 3.1 & $\mathbf{\pm 4 . 3}$ & \pm 3.8 & \pm 4.2 \\
\hline
\end{tabular}

Statistical relationships between physico-chemical and macrozoobenthos were presented in the Figs 1-12. Relationship between water temperature and benthos and current velocity and benthos was observed negative and for rest parameters it was positive.

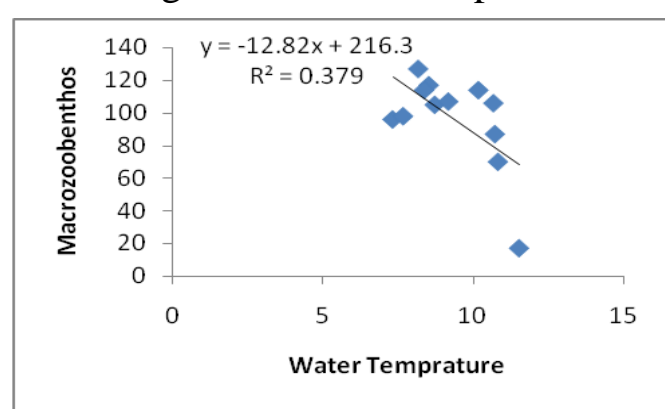

Fig 1 : Regression between Temperature and Benthos (S1)

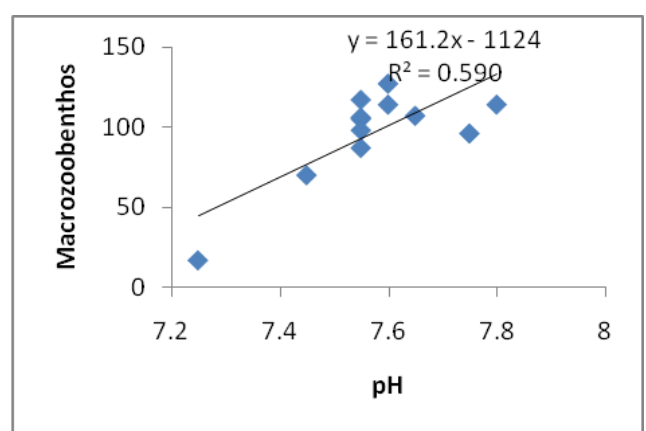

Fig 3 : Regression between $\mathrm{pH}$ and Benthos (S1)

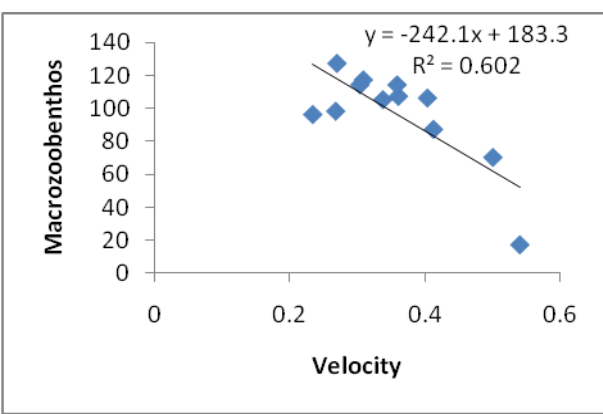

Fig 2 : Regression between velocity and Benthos (S1)

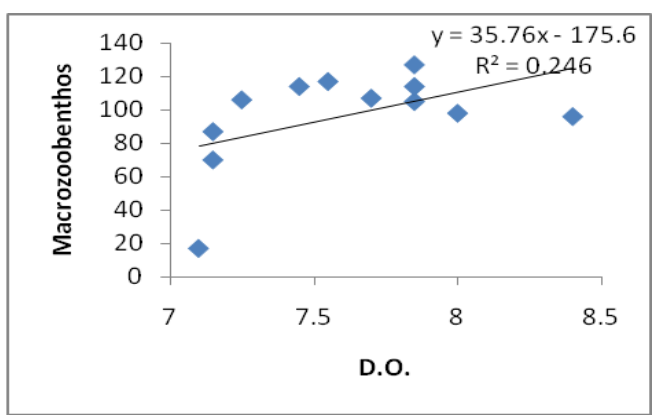

Fig 4 : Regression between DO and Benthos (S1) 


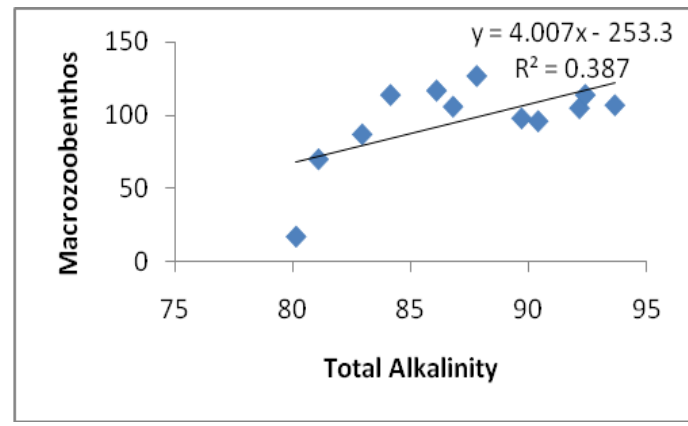

Fig 5 : Regression between TA and Benthos (S1)

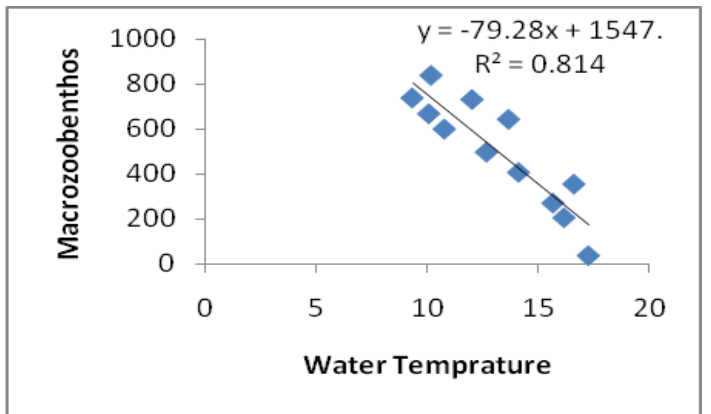

Fig 7 : Regression between Temperature and Benthos (S2)

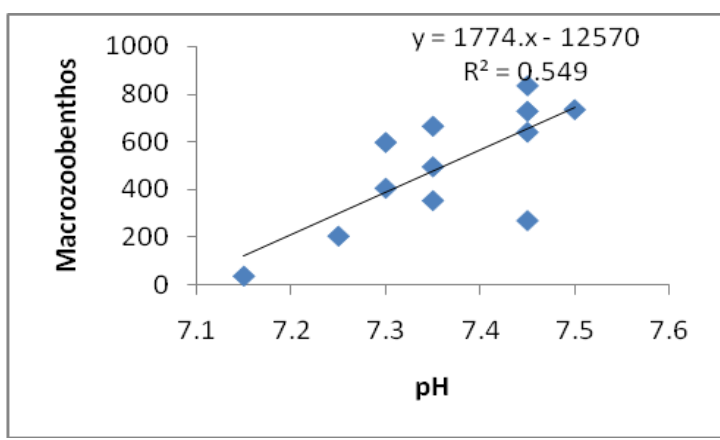

Fig 9 : Regression between $\mathrm{pH}$ and Benthos (S2)

(S2)

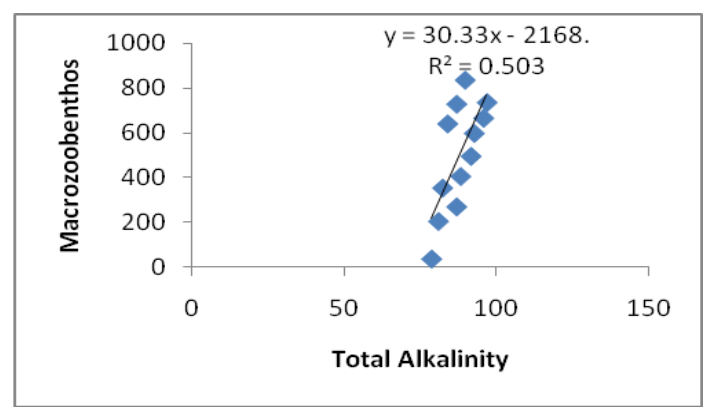

Fig 11 : Regression between TA and Benthos (S2)

Low density (17 individules. $\mathrm{m}^{-2}$ in August) and diversity (12 genus) of macrozoobenthos at Spot 1 may be due to the prevailing substrate conditions (domination by sand, gravel substrate, big bolder,

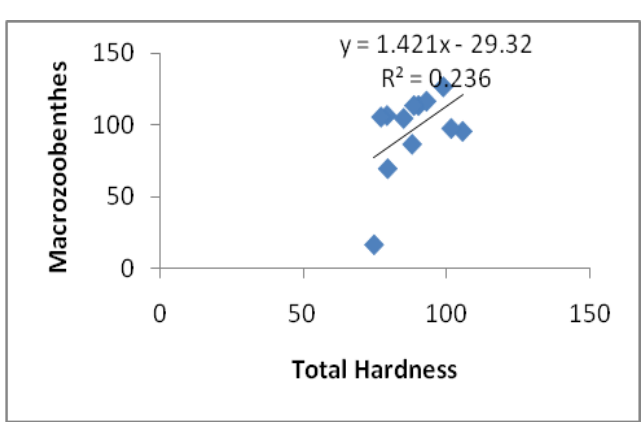

Fig 6 : Regression between TH and Benthos (S1)

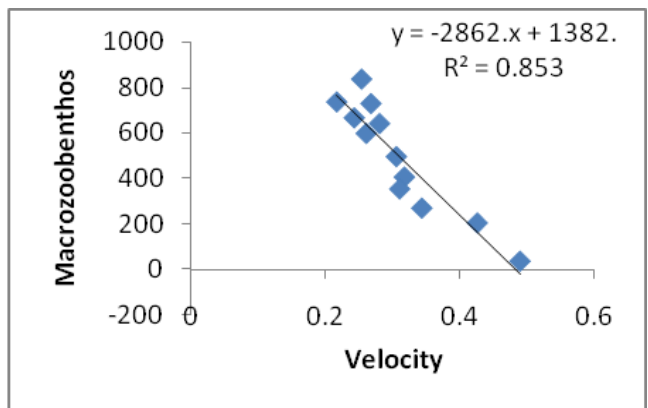

Fig 8 : Regression between velocity and Benthos (S2)

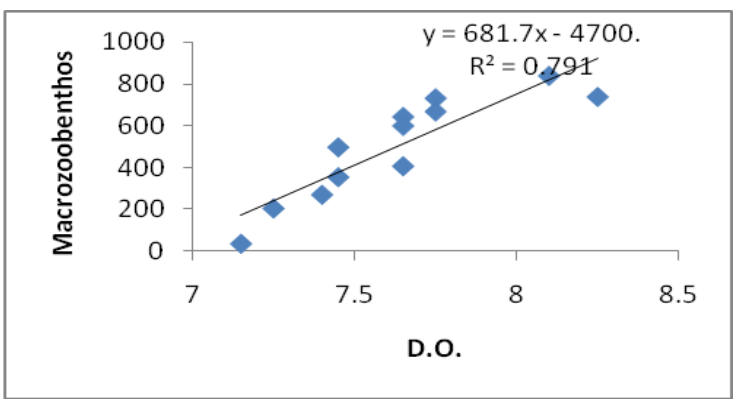

Fig 10 : Regression between DO and Benthos

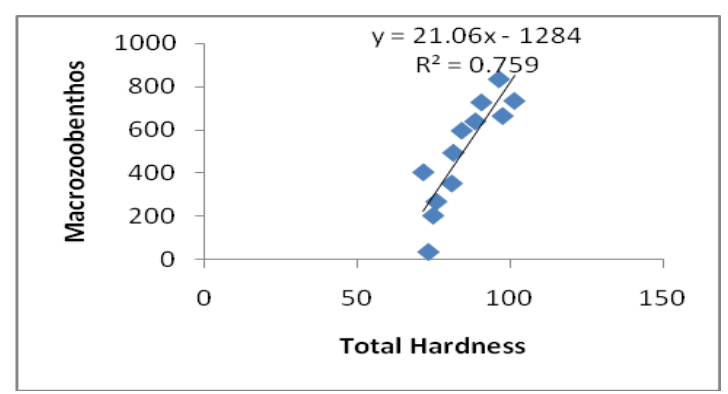

Fig 12 : Regression between TH and Benthos (S2)

faster water current, low water temperature, dense canopy and low periphytonic bottom in the stream. At Spot-2, the higher density (836 individules. $\mathrm{m}^{-2}$ ) and diversity (28 genus) of 
macrozoobenthos were perhaps due to better substratum heterogeneity dominated by mixed forest, small gravels, small bolder, low water velocity, high water temperature, low canopy of stream area and good survival rate of periphyton on bottom in the stream. During the present observation, it was noticed that density is highest in winter season (321 individules. $\mathrm{m}^{-2}$ ) and lowest in Monsoon season (87 individules. $\mathrm{m}^{-2}$ ). Baluni et.al, (2018) noticed that the periphyton was minimum in August and maximum in January in the Khankra Gad stream. It is understood that the total alkalinity favours periphyton growth in moderately flowing mountain streams, and thereby it helps in increasing macrozoobenthos density during the winter session. Similar observations have been noticed by Kumar and Dobriyal (1996) for the macrozoobenthos diversity in various small streams in Garhwal region.

The density of a species is also influenced by several ecological factors such as adaptability, substrates that support for life, and other natural factors (Welch, 1952). The nature of substratum has a significant impact on the composition and distribution of the life surviving on it (Odum 1993). Kumar et. al. (1998) stated that the macrozoobenthic density can be influenced by the heterogeneity in substratum. The number of species and their diversity changes depends on the ecological factors, such as the substratum, the depth of water, water current speed, and food availability (Kiss, 2002). When compared to structural simple substrate, such as a sand and bedrock, the physical substrate type (leaves, gravel, wood and macrophytes) generally support more diversity (Angradi, 1996; Hawkins, 1984; Baluni, et.al.2017). This can be a good explanation for the high abundance and diversity of macrozoobenthos at sampling spot-2 in Basti Damaar Gad, which has good macrophyte growth. The water temperature and current velocity had a negative correlation with zoobenthic density and total alkalinity, D.O., $\mathrm{pH}$ and total hardness demonstrated a positive relationship. Rai et al. (2019) found that $\mathrm{pH}$ and DO were the two most important variables explaining the variation in macroinvertebrate assemblages. The biotic environmental factors that influence the presence of macrozoobenthos in river cannot be separated from the ability of the species to compete in selfdefense - in this case fighting over food availability and dominating the territorial area. It is said that if a type of macrozoobenthos can be controlled or dominated in a community, the level of diversity in the community is low. On the contrary, if the level of diversity in the community is high, the dominance of existing species is low. Thus, the level of species diversity may also be influenced by the degree of dominance of species present at the station. Based on the measurement, the abiotic factors of spring fed stream maintain that physico-chemical conditions are different in each measurement of the sampling spots. These conditions correspond to the station where macrozoobenthos is located; it is due to the aquatic ecosystem of the stream that is an integral component of the set of abiotic (physicalchemical) and biotic (living organisms) environment which are related and interact with each other to form a functional structure.

The Shannon-Wiener index showed comparatively highest value during favorable December and January months and minimum during unfavorable July and August months on both spots. Similar observations were noticed in Kyunja Gad stream from Garhwal region by Bahuguna and Negi (2018).

\section{Acknowledgment}

Authors (D. M. \& P.B.) gratefully acknowledge the financial assistance rendered by Science and Engineering Research Board (SERB) as a major Project F. No.- ECR/2016/001291.

\section{References}

Abida S, Mir M F, Ifshana S, Mir S A and Ahangar I A (2012). Macrozoobenthic community as biological indicators of pollution in river Jhelum, Kashmir. Universal J Env. Res. Tech., . 2(4): 273-279.

Akindele E O and Liadi A A (2014). Diversity and response of benthic macroinvertebrates to 
Natural and Induced Environmental Stresses in Aiba Stream, Iwo, Southwestern Nigeria. West Afr. J. Appl. Ecol., 22(1): 101-111.

Angradi T R (1996). Inter-habitat variations in benthic community structure function and organic matter storage in 3 Appalachian headwater streams. J. Am. Benthos. Soc., 15: 42-63.

APHA (2005). Standard methods for examination of water and wastewater. 21st Edition., Washington D.C.

Bahuguna $\mathrm{P}$ and Dobriyal AK (2018). First report on the occurrence of some benthic macroinvertebrates in the spring fed streams of Garhwal Himalaya, India. I.J.A.R.Vol.5 (4): 437- 443.

Bahuguna P and Negi S (2018). Distribution pattern of benthic macroinvertebrate community in the spring fed stream of Garhwal Himalaya, india. J. Mountain. Res. Vol. (13):51-58.

Bahuguna $\mathrm{P}$ and Joshi $\mathrm{H} \mathrm{K}$ and Kumar $\mathrm{K}$ (2019).A report on drifting behaviour of odonata (aquatic insects) in Kyunja gad, a spring fed tributary of River Mandakini, Chamoli Garhwal, Uttarakhand. J. Mountain. Res. Vol. (14): 61-65.

Bahuguna P, Rana K K, Rayal R and Joshi H K (2020).Studies on the drifting behavioural patterns of macrozoobenthos in Kyunja Gad, a mountain stream from Garhwal Himalaya, India. J. Mountain. Res. Vol. (15): 97-108.

Balodi, VP and Koshal Kumar (2015). MacroZoobenthic study in relation to physicochemical parameters of Khoh River in Uttarakhand. Int J Sci Res, 4(6): 171-173.

Baluni P, Kumar K and Joshi H K (2018). Ecology, Distribution Pattern, Density and Diversity of Periphyton in Khankra Spring Fed Stream of Garhwal Himalaya, India. J. Mountain Res. 12: 73-79.

Baluni P, Kumar R, Chamoli KP and Joshi H K (2017). Studies on the periphyton density, diversity and physico-chemical parameters of Laster Gad stream in district Rudraprayag from India. J. Mountain Res. 12: 73-79.
Barbour MT, Gerritsen J, Griffith GE, Frydenborg R, McCarron E, White J S, Bastian M. L. (1996). A framework for biological criteria for Florida streams using benthic macroinvertebrates. J. North. Am. Benthol. Soc., 15: 185-211.

Bouchard RW and Paul Jr S (2012). Guide of aquatic Invertebrate families of Mongolia identification manual for students, citizen monitors and aquatic resources professionals. Minnesota, USA: 1-274.

Camara I A, Diomande D, Bony Y K, Ouattara A, Franquet E, Gourene G. (2012). Diversity assessment of benthic macroinvertebrate communities in Banco National Park (Banco Stream). Afr. J. Ecol., 50: 205-217.

Dobriyal AK, Balodi VP, Joshi HK, Thapliyal A, Bahuguna Pankaj, Uniyal SP and Kotnala CB (2009). Substratum heterogeneity and indicator macrozoobenthos of the Eastern Nayar, Garhwal, Central Himalaya. $J$. Mountain. Res. Vol. (4): 130-135.

Dobriyal AK (1985). Ecology of limnofauna in small streams and their importance to the village life in Garhwal Himalaya. Uttar Pradesh J. Zool. 5 : 139- 144.

Dobriyal AK, Balodi VP, Joshi HK. and Bahuguna Pankaj (2011). Seasonal cycle city of macrozoobenthos correlated with detrimental abiotic factor in Eastern Nayar of Garhwal Himalaya, Uttarakhand. (In." Aquatic Biodiversity "), (Edt. By. Madhu Thapliyal and Ashish Thapliyal): "Transmedia Publication".: 94-103

Elliot J M, Humpesch U H and Macan T T. (1988) Larvae of the British Ephemeroptera, a key with ecological notes. Scientific Publication of the Freshw Biol. Assoc., 49:145.

Hawkins C. P. (1984). Substrate association and longitudinal distribution in species of Ephemerellidae (Ephemeroptera:Insect) from Western Oregon. Freshw Inver. Biol., 3 (94): 18-88.

Hynes HBN. (1977) A key adults and nymphs of the Britisch stoneflies (Plecoptera) with notes on their ecology and distribution. Freshwater 
Biological Association, ambleside, Scientific Publication 17.

Jacobson D, Rostgaard S. and Vasconez J. J. (2003). Are macroinvertebrates in high altitude streams affected by oxygen deficiency? Freshw. Biol. 48(11): 2025-2032.

Joshi P C, Negi R K, Negi T (2007) Seasonal variation in benthic macro-invertebrates and their correlation with the environmental variables in a freshwater stream in Garhwal region (India). Life Sci. J 4:85-93.

Kabore I, Moog O, Alp M, Guenda W, Koblinger T, Mano K, Oueda A, Ouédraogo R, Trauner D. and Melcher A. H. (2016). Using macroinvertebrates for ecosystem health assessment in semi-arid streams of Burkina Faso. Hydrobiologia 766(1): 57-74.

Kiss O. (2002). Trichopteran communities of a rill and a stream in the Bukk Mts. (North Hungary). Nova Suppl. Ent. Vol. 15. P. 53543.

Kumar N. and Dobriyal A. K. (1996). Benthic diversity of Garhwal Himalayan hillstreams in relation to their fishery potential. In: Joseph, M. M. (Ed.) The Third Indian fisheries Forum Proceedings.p.159-162.

Kumar N, Kumar B. and Dobriyal A. K. (1998). Geomorphic influences on the distribution of benthic diversity in two hillstreams of Garhwal Himalaya. In: Johal, M.S. (Edited): Proceedings of Indo-US Workshop on Conservation and Development of Natural Fishery Resources of Western Himalayas. Pp. 53 - 66.

Koshal Kumar, Jitendra Singh Rana, Anita Rawat Rana and Kotnala, CB (2017) Checklist of Benthic Macroinvertebrate Communities of Stream Rawasan In Garhwal Region (Central Himalaya), Uttarakhand (India). J Mountain Res 12: 91-95.

Marzin A. (2013). Ecological assessment of running water using bio-indicator: association variability and uncertainty. Doctorate thesis agros Paris Tech.: 1-202pp.

Nautiyal P, Mishra A S, Semwal V P. (2015). Spatial distribution of benthic macroinvertebrate fauna in mountain streams of Uttarakhand, India. In: M. Rawat et al. (eds.), Aqu. Ecos. Biodi. Ecol. Conv.,Pp. 3151 Springer India.

Needham I. G. and Needham P. R. (1962). A guide to the study of freshwater biology. San Francisco Holden Day Inc. 108 PP.

Negi RK. and Mamgain S (2013). Seasonal variation of benthic macro invertebrates from Tons river of Garhwal Himalaya Uttarakhand. Pakistan J Biol. Sci.,16 (22): 1510-1516.

Odum EP (1993). Fundamental of ecology. Yogyakarta. Gajah Mada University Press.

Rai A, Shah DN, Shah RDT and Milner C (2019). Influence of environmental parameters on benthic macroinvertebrate assemblages in the headwaters of Bagmati river, Kathmandu valley, Nepal. Banko Janakari 29 (1):53-61.

Resh V H, Brown A V, Covich APME, Li HW. and Minshall G.W.(1988) The role of disturbance in stream ecology. J. N. Am. Benthol. Soc. 7:33-455.

Rosenberg D and Resh VH. (1993). Freshwater biomonitoring and benthic invertebrates (pp. 1-448). New York: Chapman and Hall.

Sagir M, Rashid M, Bahuguna, P. and Dobriyal A $K$ (2018). Impact of riparian vegetation on the structure and function of Nayer river ecosystem. J. Mountain. Res. Vol.13: 21-28.

Sharma RC, Bhanot G and Singh D. (2004). Aquatic macroinvertebrate diversity in Nanda Devi Biosphere Reserve, India. Env.. 24: 211-221

Sharma S, Dubey S and Chaurasia R (2013). Benthic macro invertebrate and its correlations with physico-chemical parameters from Kunda river, Khargone (M.P.), India. Int. J Adv Res. 1(2): 8-13.

Silveira M.P, Buss DF, Nessimian J L. and Baptista D F (2006). Spatial and temporal distribution of benthic macroinvertebrates in a Southeastern Brazilian river. Braz. J. Biol.66 (2B):623-632.

Sivaramkrishnan K.G, Venkataraman K, Sridhar S. and Marinuthu U.(1995). Spatial patterns of benthic macroinvertebrate distribution 
along river kaveri and its tributaries (India).

Int. J Ecol. Envir. Sci. 21:141-161.

Sunder S (1997). Biotic communities of a Kumaon Himalayan River-The Gaula - 1 . Macrobenthic invertebrates. Proc. Nat. Acad. Sci. India, Sect B Biol Sci 67 (B) II:157-167.

Uwadiae R E (2013). Composition, Distribution and Diversity of Benthic Macroinvertebrates of Ona River, Southwest, Nigeria. Eur. J. Zool. Res., 1(2):47-53.

Vinson M R and Hawkins C P (1998). Biodiversity of stream insects: variations at local basin and regional scales. Annual Rev. Entmo., 43, 21-293.

Wallace I D, Wallace B and Philipson G N (2003). Keys to the case bearing caddies larvae of Britain and Ireland. Fresh water Biol. Asso.:1-259.

Ward H B and Whipple G V (1959). Freshwater Biology (ed.W. T. Edmondson). J. Wiley and sons. Inc. New York 1203 pp.

Ward J V and Tockner K (2001). Biodiversity: Towards a unifying theme for river ecology. Freshw Biol., 46, 807-819.

Welch P S (1952). Limnology. Mc Graw-Hill Book Co. Inc. New York.

$* * * * * * *$ 